\title{
Gamapatias Monoclonais de Significado Indeterminado: Critérios de Diagnóstico e Acompanhamento Clínico
}

\author{
Monoclonal Gammopathies of Indetermined Significance: Diagnosis and \\ Clinical Follow-up Guidelines
}

\author{
Joana PARREIRA ${ }^{1}$, Paulo LÚCIO², Cristina JOÃO ${ }^{3}$, Ana MACEDO ${ }^{4,5}$, Ana Bela SARMENTO ${ }^{6,7}$, Catarina GERALDES ${ }^{6,7}$, \\ Cristina GONÇALVES ${ }^{8}$, Graça ESTEVES ${ }^{9}$ \\ Acta Med Port 2014 Sep-Oct;27(5):661-664
}

\section{RESUMO}

O Grupo Português de Mieloma Múltiplo da Sociedade Portuguesa de Hematologia propôs-se criar um protocolo de avaliação e acompanhamento de doentes com gamapatias monoclonais. O protocolo aqui apresentado, meramente referencial, pretende racionalizar e uniformizar a forma como estes doentes são acompanhados pelos seus médicos assistentes e identifica critérios claros de risco de progressão que justificam a sua referenciação a uma consulta de Hematologia Clínica.

Palavras-chave: Gamapatia Monoclonal de Significado Indeterminado; Prognóstico; Progressão da Doença; Portugal.

\section{ABSTRACT}

The Portuguese group of multiple myeloma of the Portuguese Society of Hematology proposes a national protocol for diagnosis and clinical follow-up of monoclonal gammopathies. The proposed protocol aims to standardize clinical management of monoclonal gammopathies. Furthermore, it would also define the major risk factors for progression to Multiple Myeloma that require a precocious close articulation between general practitioners and a Hematology Clinic.

Keywords: Monoclonal Gammopathy of Undetermined Significance; Prognosis; Disease Progression; Long-Term Care; Portugal.

\section{INTRODUÇÃO}

As gamapatias monoclonais constituem um grupo de entidades clínicas associadas à proliferação monoclonal de plasmócitos e caracterizam-se pela existência de uma imunoglobulina clonal (paraproteína) no soro e/ou urina. Para que seja considerada gamapatia monoclonal de significado indeterminado (GMSI) o pico monoclonal no sangue periférico deverá ser inferior a $3 \mathrm{~g} / \mathrm{dL}$, a proteína de Bence-Jones na urina de 24 horas inferior a $0,5 \mathrm{~g} / \mathrm{dL}$ e a plasmocitose medular inferior a 10\%. São ainda critérios necessários para o diagnóstico de GMSI a ausência de envolvimento de órgãos alvo (ossos, rim e medula óssea) e a ausência de evidência clínica de mieloma múltiplo (MM), macroglobulinemia de Waldenstrom, linfoma ou amiloidose. ${ }^{1,4}$ A GMSI surge em cerca de $3 \%$ de indivíduos com mais de 50 anos, podendo esta incidência ultrapassar os $5 \%$ na população com idade superior a 70 anos. $^{4}$

Globalmente, a GMSI tem um risco de evolução para mieloma múltiplo de $1 \%$ /ano. Por esta razão, é necessária a vigilância regular dos indivíduos a quem se detecta um pico monoclonal, de forma a antecipar a progressão para doença sintomática e, desta forma, evitar as potenciais complicações resultantes da lesão de órgãos alvo (MM sintomático). ${ }^{3,8}$ Este risco não é igual em todos os casos, sendo importante proceder à sua estratificação na data do diagnóstico.

Classicamente consideradas numa só entidade, as GMSI podem subdividir-se em dois grupos: linfóide (ou linfoplasmocitóide) e plasmática. ${ }^{16}$ As GMSI plasmáticas dividem-se em três subtipos clínicos, de acordo com o isotipo envolvido:

- As GMSI não $\lg M(\lg G, \lg A$, $\lg D)$, constituem o subtipo mais frequente, podem progredir para mieloma múltiplo indolente ou sintomático e mais raramente para amiloidose $A L$, doença de deposição de cadeias leves ou outra doença linfoproliferativa;

- As GMSI IgM representam cerca de $15 \%$ dos casos, apresentam risco de progressão para macroglobulinemia de Waldenstrom e mais raramente linfoma ou amiloidose AL. Raramente podem progredir para mieloma múltiplo IgM.

- As GMSI de cadeias leves constituem o subtipo mais raro de GMSI, apresentam risco potencial de progressão para mieloma múltiplo a cadeias leves, amiloidose AL ou doença de cadeias leves livres.

Uma vez que a maioria dos doentes com GMSI é identificada e acompanhada em consultas de medicina geral ou por especialidades que não a Hematologia, o Grupo Por-

\footnotetext{
1. Serviço de Hematologia. Instituto Português de Oncologia Francisco Gentil. Faculdade de Medicina. Universidade de Lisboa. Lisboa. Portugal.

2. Unidade de Hemato-Oncologia. Fundação Champalimaud. Lisboa. Portugal.

3. Serviço de Hematologia. Instituto Português de Oncologia Francisco Gentil. Faculdade de Ciências Médicas. Universidade de Lisboa. Lisboa. Portugal.

4. Serviço de Hematologia. Centro Hospitalar do Algarve. Faro. Portugal.

5. Faculdade de Ciências da Saúde. Universidade da Beira Interior. Covilhã. Portugal.

6. Serviço de Hematologia Clínica. Centro Hospitalar Universitário de Coimbra. Coimbra. Portugal.

7. Centro de Investigação em Meio Ambiente Genética e Oncobiologia. Faculdade de Medicina. Universidade de Coimbra. Coimbra. Portugal

8. Serviço de Hematologia Clínica. Hospital de Santo António. Porto. Portugal.

9. Serviço de Hematologia. Hospital de Santa Maria. Centro Hospitalar de Lisboa Norte. Lisboa. Portugal.

Recebido: 23 de Abril de 2014 - Aceite: 14 de Julho de 2014 | Copyright @ Ordem dos Médicos 2014
} 
tuguês de Mieloma Múltiplo (GPMM) propôs-se elaborar um conjunto de sugestões para o correcto diagnóstico e acompanhamento de gamapatias monoclonais que respeite o estado da arte e que garanta aos doentes um correcto acompanhamento clínico até à sua eventual progressão e referenciação a uma consulta da especialidade.

\section{OBJECTIVOS}

Foram objectivos deste grupo criar um protocolo de avaliação inicial e acompanhamento de doentes com gamapatias monoclonais, assim como identificar critérios claros de risco de progressão que justifiquem a referenciação destes doentes à consulta de Hematologia Clínica.

\section{Avaliação inicial:}

A avaliação laboratorial inicial de um doente com gamapatia monoclonal deve incluir a realização das seguintes análises:

- Hemograma com contagem de plaquetas (em caso de existência de linfocitose ou linfócitos atípicos deverá ser feita imunofenotipagem de sangue periférico por citometria de fluxo),

- Função renal, doseamento de cálcio sérico, doseamento de B2-microglobulina e LDH,

- Imunofixação, proteinograma, doseamento sérico de imunoglobulinas (se imunofixação sérica negativa, procurar os subtipos de imunoglobulinas $\lg \mathrm{D}$ e $\lg \mathrm{E}$ ),

- Doseamento das cadeias leves livres no soro,

- Urina tipo II,

- Doseamento de proteína Bence-Jones na urina de 24h,

- Avaliação medular - está indicada em todos os casos com paraproteína $\geq 1,5 \mathrm{~g} / \mathrm{dL}$, casos de gamapatia monoclonal não IgG ou em casos de anomalias no hemograma, creatinina sérica, cálcio ou alterações na avaliação do esqueleto. A avaliação medular deverá ser considerada em todos os doentes com suspeita clínica de doença linfoproliferativa ou mieloma múltiplo, e em indivíduos com GMSI e idade inferior a 65 anos. ${ }^{9} \mathrm{~A}$ avaliação medular deve incluir:

- Mielograma: permite avaliar o grau de infiltração por plasmócitos da medula óssea.

- Biopsia óssea: essencial na suspeita de doença linfoproliferativa e MM.

- Imunofenotipagem da medula óssea por citometria de fluxo: permite a detecção de plasmócitos com fenótipo aberrante e sua quantificação e a avaliação do índice de ploidia dos plasmócitos.

- Estudo citogenético dos plasmócitos por FISH: pesquisa de $t(4 ; 14)$, del $17 p 13, t(11 ; 14), t(14 ; 16)$ e anomalias do cromossoma 1.

Os estudos imunofenotípico e citogenético da medula óssea implicam o acesso a laboratórios diferenciados no estudo de doenças hemato-oncológicas e não têm impacto imediato no acompanhamento das GMSI. Em contrapartida, são estudos de grande importância prognóstica em caso de progressão para MM. Neste contexto, a sua realização deverá ser discutida com um hematologista e ponderada caso a caso em função do risco de progressão, da acessibilidade aos laboratórios referidos e dos custos envolvidos.

A avaliação imagiológica do esqueleto, para exclusão de lesões osteolíticas, inclui:

- Radiografia simples do esqueleto ou, preferencialmente, Tomografia Computorizada (TC) do esqueleto sem contraste. Em caso de lesões suspeitas é aconselhada a realização de RMN da coluna vertebral;

- Ecografia abdominal / TC cervical-tóraco-abdomino-péIvica, para avaliar presença de adenomegalias ou organomegalias, em caso de gamapatia IgM ou suspeita de doença linfoproliferativa.

Estabelecido o diagnóstico de gamapatia monoclonal, é necessária a distinção entre GMSI e Mieloma Múltiplo, atendendo aos critérios já citados - pico sérico inferior a $3 \mathrm{~g} / \mathrm{dL}$, infiltração medular por plasmócitos inferior a 10\%, ausência de lesões ósseas, anemia, hipercalcemia ou insuficiência renal (os clássicos sintomas $C R A B$ ), relacionadas com a paraproteína e exclusão de doença linfoproliferativa (Tabela 1).

\section{Avaliação do risco de progressão das GMSI}

Reconhecida a GMSI, é importante identificar factores indicativos de maior risco de progressão para Mieloma e assim estratificar grupos prognósticos. ${ }^{2,7,8}$ Das diferentes propostas existentes na literatura, os sistemas de estratificação propostos pelo grupo cooperativo espanhol PETHEMA e pela Clínica Mayo são os que apresentam maior evidência científica.

A proposta do grupo espanhol baseia-se na caracterização imunofenotípica dos plasmócitos e na razão entre plasmócitos normais e patológicos na medula óssea. Esta abordagem exige o apoio de uma citometria de fluxo sofisticada, cuja reprodutibilidade entre diferentes laboratórios ainda carece de demonstração. ${ }^{17}$

O grupo da Clínica Mayo define como factores de ris$\mathrm{CO}:^{-1,12-14}$

1. $O$ isotipo de imunoglobulina $(\lg M$ ou $\lg A)$,

2. A quantidade de proteína monoclonal (superior a $1,5 \mathrm{~g} /$ $\mathrm{dL})$

3. A relação entre as cadeias leves livres $(<0,26$ ou $>$ $1,65)$.

Atendendo ao facto das variáveis utilizadas pela Clínica Mayo serem facilmente quantificáveis e à reprodutibilidade dos resultados obtidos, o GPMM propõe que a estratificação de risco de progressão seja efectuada recorrendo a estes critérios.

De acordo com a estratificação proposta, o risco absoluto de progressão de GMSI para Mieloma Múltiplo ao longo de 20 anos para doentes com diferentes combinações de factores de risco, está representado na Tabela 2.1,5,7 
Tabela 1 - Critérios de diagnóstico de GMSI, MMI e MM

Critérios IMWG, 2010 5,18

GMSI Proteína M sérica $<3 \mathrm{~g} / \mathrm{dL}$, e plasmócitos clonais na medula óssea $<10 \%$ e

Ausência de lesão de órgão alvo*

Presença de proteína $\mathrm{M}$ sérica e/ou urinária $\geq 3 \mathrm{~g} / \mathrm{dL}$ e/ou

MMI Plasmócitos clonais na medula óssea $\geq 10 \% \mathrm{e}$

Ausência de lesão de órgão alvo*

Qualquer valor de proteína M sérica e/ou urinária excepto nos casos de mieloma não secretor**.

Plasmócitos clonais na medula óssea $\geq 10 \%$ ou em biópsia de tecido.

IMWG2003. ${ }^{18}$ diagnóstico baseado na identificação de sinais de lesão de órgão alvo (CRAB):

- Hipercalcemia (cálcio sérico $>11,5 \mathrm{mg} / \mathrm{dL}$ )

- Insuficiência renal (creatinina $>1,95 \mathrm{mg} / \mathrm{dL}$ sem outra etiologia)

MM

- Anemia (hemoglobina $<10 \mathrm{~g} / \mathrm{dL}$ ): normocrómica e normocítica com valor de $\mathrm{Hb}>2 \mathrm{~g} / \mathrm{dL}$ abaixo do limite inferior do normal

- Lesões do esqueleto (lesões líticas por radiografia do esqueleto, osteoporose com fracturas patológicas, ou compressão medular)

Critérios adicionais incluem:

- Infecções bacterianas recurrentes (> 2 em 12 meses)

- Amiloidose

- Hiperviscosidade sintomática

GMSI: gamapatia monoclonal de significado indeterminado; MMI: mieloma múltiplo indolente; MM: mieloma múltiplo.

*qualquer um dos seguintes: hipercalcemia $>11,5 \mathrm{mg} / \mathrm{dL}$, insuficiência renal com creatinina $>2,0 \mathrm{mg} / \mathrm{dL}$, ou depuração da creatinina estimada $<40 \mathrm{ml} / \mathrm{min}$, anemia normocrómica e normocítica com $\mathrm{Hb}<10 \mathrm{~g} / \mathrm{dL}$ (ou valor de $\mathrm{Hb} 2 \mathrm{~g} / \mathrm{dL}$ < limite inferior do normal) e lesões ósseas (lesões líticas, osteopenia grave ou fractura patológica).5,18

** Mais que $10 \%$ de plasmócitos é necessário para o diagnóstico de mieloma não secretor.

Tabela 2 - Modelo preditivo de estratificação do risco de progressão de GMSI para MM ao longo de 20 anos

\begin{tabular}{lcc}
\hline Grupo de risco & $\mathbf{N}^{\circ}$ de factores* & Risco absoluto de progressão (\%) \\
\hline Alto risco & 3 & $58 \%$ \\
Risco Intermédio-alto & 2 & $37 \%$ \\
Risco Intermédio-baixo & 1 & $21 \%$ \\
Baixo risco & 0 & $5 \%$ \\
${ }^{*}$ o isotipo de imunoglobulina (IgM ou $\left.\lg \mathrm{A}\right)$, a quantidade de proteína monoclonal (superior a 1,5 g/dL), e a relação entre as cadeias leves livres $(<0,26$ ou > 1,65).
\end{tabular}

Recomendações para o Acompanhamento de Doentes com GMSI:

GMSI de baixo risco (cerca de $50 \%$ de todos os casos de GMSI): Deverão ter um acompanhamento semestral durante o primeiro ano, podendo posteriormente passar a ser monitorizadas de dois em dois anos. Este acompanhamento poderá ser feito pelo médico assistente e deve incluir:

- Hemograma com contagem de plaquetas,

- Função renal, doseamento de cálcio sérico, doseamento de B2-microglobulina e LDH,

- Imunofixação, proteinograma e doseamento sérico de imunoglobulinas,

- Doseamento das cadeias leves livres no soro,

- Urina tipo II, Doseamento de proteína Bence-Jones na urina de $24 \mathrm{~h}, \mathrm{Rx}$ do esqueleto/TC ou RM, se dores ósseas.

GMSI de risco intermédio ou alto: Deverá ser equacionada a referenciação destes doentes a uma consulta de Hematologia. A avaliação laboratorial é sobreponível ao descrito anteriormente mas deverá ser mais frequente e poderá ser condicionada pelo aparecimento de sintomatologia suspeita de progressão para mieloma múltiplo. A sua frequência dependerá da evolução do pico monoclonal e da existência ou não de sintomas. Após um acompanhamento inicial de três em três ou de quatro em quatro meses, desde que a situação esteja estável, a vigilância poderá passar a semestral nos primeiros dois anos e posteriormente anual, se não se verificar progressão. Dado o maior risco de progressão, o GPMM recomenda a avaliação medular inicial destes doentes e a vigilância imagiológica da doença óssea independentemente da existência ou não de sintomas suspeitos. A repetição de exames de imagem para avaliação de alterações esqueléticas, deverá ser orientada pelos sintomas, nomeadamente aparecimento de dor óssea de novo ou claro agravamento do pico monoclonal. ${ }^{9}$

\section{CONCLUSÕES}

É cada vez mais importante a uniformização do acompanhamento de diversas entidades clínicas, especialmente quando se tratam de situações pré-malignas. Nestas situações, uma vigilância adequada poderá permitir a detecção precoce de transformação maligna e consequentemente a intervenção terapêutica mais rápida.

A GMSI é uma entidade nosológica com um risco significativo de evolução para Mieloma Múltiplo. Este risco jus- 
tifica o acompanhamento regular dos doentes com GMSI mas, de acordo com a experiência clínica dos autores, este acompanhamento é actualmente feito de forma muito heterogénea. A definição de um protocolo de acompanhamento clínico e laboratorial, permite optimizar a gestão dos recursos envolvidos neste acompanhamento (consultas médicas, exames analíticos e imagiológicos); a avaliação da probabilidade de evolução para Mieloma Múltiplo permite, de forma objectiva, clara e acessível, identificar precocemente os doentes de pior prognóstico que beneficiam do acompanhamento em consulta de Hematologia.

\section{REFERÊNCIAS}

1. Rosiñol L, Cibeira MT, Montoto S, Rosman M, Esteve J, Filella X, et al. Monoclonal gammopathy of undetermined significance: predictors of malignant transformation and recognition of an evolving type characterized by a progressive increase in $\mathrm{M}$ protein size. Mayo Clin Proc. 2007;82:428-43.

2. Rajkumar SV, Kyle RA, Therneau TM, Melton LJ, Bradwellet AR, Clark $\mathrm{RJ}$, et al. Serum free light chain ratio is an independent risk factor for progression in monoclonal gammopathy of undetermined significance. Blood. 2005;106:812-7.

3. Rajkumar SV. MGUS and smoldering multiple myeloma: update on pathogenesis, natural history, and management. Hematology. 2005; $1: 340-5$.

4. Kyle RA, Therneau TM, Rajkumar SV, Larson DR, Plevak MF, Offord JR, et al. Prevalence of monoclonal gammopathy of undetermined significance. N Engl J Med. 2006;354:1362-9.

5. Kyle RA, Durie BG, Rajkumar SV, Landgren O, Blade J, Merlini G, et al. Monoclonal gammopathy of undetermined significance (MGUS) and smoldering (asymptomatic) multiple myeloma: IMWG consensus perspectives risk factors for progression and guidelines for monitoring and management. Leukemia. 2010;24:1121-7.

6. Kyle R, Rajkumar SV. Multiple myeloma. Blood. 2008;111:2962-72.

7. Rajkumar SV, Kyle RA, Buadi FK. Advances in the diagnosis, classification, risk stratification, and management of monoclonal gammopathy of undetermined significance: implications for recategorizing disease entities in the presence of evolving scientific evidence. Mayo Clin Proc. 2010;85:945-8.

8. Dispenzieri A, Katzmann JA, Kyle RA, Larson DR, Melton LJ, Colby $\mathrm{CL}$, et al. Prevalence and risk of progression of light-chain monoclonal gammopathy of undetermined significance: a retrospective populationbased cohort study. Lancet. 2010;375:1721-8.

9. Berenson JR, Anderson KC, Audell RA, Boccia RV, Coleman M, Dimopoulos MA, et al. Monoclonal gammopathy of undetermined significance: a consensus statement. Br J Haematol. 2010;150:28-38.

10. Bianchi G, Kyle RA, Colby CL, Larson DR, Kumar S, Katzmann JA, et al.

\section{CONFLITOS DE INTERESSE}

Sem conflitos de interesse a declarar. Os autores declaram integrar o Grupo Português de Mieloma Múltiplo da Sociedade Portuguesa de Hematologia.

\section{FONTES DE FINANCIAMENTO}

Este trabalho não recebeu qualquer contribuição, subsídio ou bolsa.

Impact of optimal follow-up of monoclonal gammopathy of undetermined significance on early diagnosis and prevention of myeloma-related complications. Blood. 2010;116:2019-25.

11. Dimopoulos M, Kyle R, Fermand JP, Rajkumar SV, San Miguel J, Chanan-Khan A, et al. Consensus recommendations for standard investigative workup: report of the International Myeloma Workshop Consensus Panel 3. Blood. 2011;117:4701-5.

12. Kyle RA, Therneau TM, Rajkumar SV, Offord JR, Larson DR, Plevak $\mathrm{MF}$, et al. A long-term study of prognosis in monoclonal gammopathy of undetermined significance. N Engl J Med. 2002;346:564-9.

13. Baldini L, Guffanti A, Cesana BM, Colombi M, Chiorboli O, Damilano I, et al. Role of different hematologic variables in defining the risk of malignant transformation in monoclonal gammopathy. Blood. 1996;87:912-8.

14. Cesana C, Klersy C, Barbarano L, Nosari AM, Crugnola M, Pungolino $\mathrm{E}$, et al. Prognostic factors for malignant transformation in monoclonal gammopathy of undetermined significance and smoldering multiple myeloma. J Clin Oncol. 2002;20:1625-34.

15. Landgren O. Monoclonal gammopathy of undetermined significance and smoldering multiple myeloma: biological insights and early treatment strategies. Hematology. 2013;1:478-87.

16. Korde N, Krintinsson SY, Kandgren O. Monoclonal gammopathy of undetermined significance (MGUS) and smoldering myeloma (SMM): novel biological insights and development of early treatment strategies. Blood. 2011;117:5573-81.

17. Perez-Persona E, Vidriales MB, Mateo G, Garcia-Sanz R, Mateos MV, de Coca AG, et al. New criteria to identify risk of progression in monoclonal gammopathy of uncertain significance and smoldering multiple myeloma based on multiparameter flow cytometry analysis of bone marrow plasma cells. Blood. 2007;110:2586-92.

18. The International Myeloma Working Group. Criteria for the classification of monoclonal gammopathies, multiple myeloma and related disorders: a report of the International Myeloma Working Group. Br J Haematol. 2003;121:749-57. 
Joana PARREIRA, Paulo LÚCIO, Cristina JOÃO, Ana MACEDO, Ana Bela SARMENTO, Catarina GERALDES, Cristina GONÇALVES, Graça ESTEVES

\section{Gamapatias Monoclonais de Significado Indeterminado: Critérios de Diagnóstico e Acompanhamento Clínico}

Acta Med Port 2014:27:661-664

Publicado pela Acta Médica Portuguesa, a Revista Científica da Ordem dos Médicos

Av. Almirante Gago Coutinho, 151

1749-084 Lisboa, Portugal.

Tel: +351218428 215

E-mail: submissao@actamedicaportuguesa.com

www.actamedicaportuguesa.com

ISSN:0870-399X | e-ISSN: 1646-0758

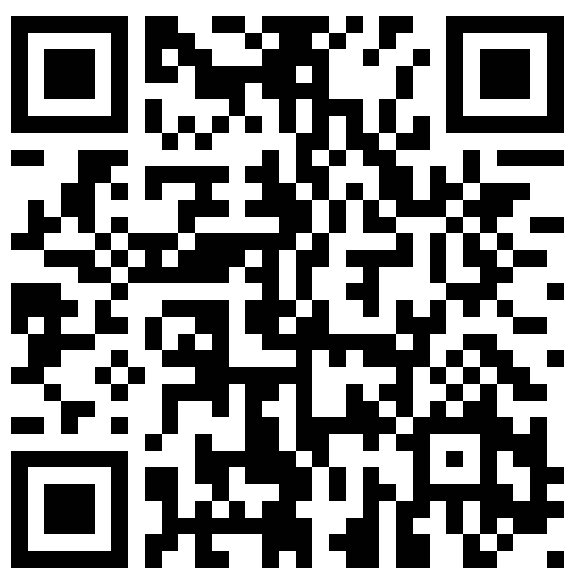

